\title{
Estudo de Alguns Fatores de Risco para a Presença de Mecônio no Líquido Amniótico
}

Autor: Octávio de Oliveira Santos Filho

Orientadora: Prof $^{a}$.Dr ${ }^{a}$. Celina de Paula Azevedo Sollero

Dissertação apresentada à Faculdade de Ciências Médicas da Pontifícia Universidade Católica de Campinas para a obtenção do Título de Mestre em Medicina do Programa de Pós-Graduação na área de Clínica Cirúrgica - Tocoginecologia em 28 de junho de 2000.

A presença de mecônio no líquido amniótico ocorre em cerca de 8 a 25\% das gestações. Existe um consenso, na obstetrícia atual, de que o líquido meconial pode comprometer o ciclo gravídico puerperal, aumentando a morbidade e a mortalidade materno-fetal. Por outro lado, a etiologia de sua passagem para o líquido amniótico permanece obscura. Alguns autores acreditam ser um fenômeno fisiológico, ao passo que para outros o fenômeno ocorre em razão de fatores de estresse fetal, e um terceiro grupo acata as duas teorias. Os resultados encontrados na literatura, em relação a fatores de risco para a presença de mecônio no líquido amniótico, não estão bem esclarecidos. Com o objetivo de identificarmos alguns fatores que tenham relação com a presença de mecônio no líquido amniótico, realizamos um estudo retrospectivo com pacientes assistidas no Hospital e Maternidade Celso Pierro da Pontifícia Universidade Católica de Campinas, no período de janeiro a dezembro de 1998. Analisamos 3158 prontuários e obtivemos uma amostra final de 465 gestantes que foram classificadas em dois grupos: o das gestantes que não apresentavam mecônio no liquido amniótico (382 pacientes) e o das que o apresentavam (83 pacientes). Utilizamos para a análise estatística métodos de regressão logística uni e multivariada e stepwise. Consideramos um $\mathrm{p} \leq \mathrm{a} 0.05$ para o nosso nível de significância. Tivemos como resultado, dentre as variáveis estudadas, o seguinte: a idade das pacientes, paridade, número de consultas pré-natais, cesárea prévia, peso ao nascer e peso placentário não tiveram influência na passagem de mecônio para o líquido amniótico. Entretanto, a idade gestacional e as alterações dos batimentos cardiacos fetais alteraram, de maneira significante, tal ocorrência. Para a variável idade gestacional, a cada semana que se avançou, tivemos uma chance de 1,37 vezes maior de termos mecônio no liquido amniótico. Quanto às gestantes com alterações nos batimentos cardíacos fetais, detectamos o índice de 5,47 vezes maior de que estas venham a apresentar mecônio no líquido amniótico. Conclusão: Os resultados deste estudo sugerem que a idade materna, paridade, cesárea prévia, número de consultas pré-natais, peso ao nascer e peso placentário não são fatores de risco para a presença de mecônio no líquido amniótico. Por outro lado, o avanço da idade gestacional e as alterações dos batimentos cardiacos fetais mostraram-se como fatores estatisticamente significantes.

Palavras-chave: Gravidez, complicações. Líquido amniótico. Mecônio. Relação com Morbidade e Mortalidade Neonatal Precoce.

\author{
Autora: Adehilde Martins Santos \\ Orientador: Prof. Dr. José Elias Soares da Rocha
}

Dissertação apresentada ao Curso de Pós-Graduação em Saúde da Criança do Departamento de Tocoginecologia e Pediatria da Universidade Federal de Alagoas (UFAL), para obtenção do Título de Mestre em 19 de julho de 2000.

O presente estudo teve como objetivo diagnosticar o crescimento intra-uterino retardado (CIUR) mediante a utilização do Índice Ponderal de Rohrer (IPR) e sua associação com morbidade e mortalidade neonatal precoce.

Pacientes e Métodos: Foram estudados 2741 recém-nascidos (RN) na Maternidade Santa Mônica da Escola de Ciências Médicas de Alagoas no período compreendido entre janeiro a dezembro de 1998. O desenho do estudo foi de coorte do tipo retrospectivo. Foram incluídos RN de termo de mulheres tabagistas (> 10 cigarros/ dia), portadoras de amniorrexe, doença hipertensiva especifica da gravidez (DHEG), formas: pré-eclâmpsia leve $(\mathrm{PEL})$, pré-eclâmpsia grave $(\mathrm{PEG})$ e eclâmpsia e de mulheres hígidas e não consumidoras de tabaco e/ ou outras drogas. O cálculo do IPR foi realizado a partir da equação proposta por Rohrer: IP $=\mathrm{P} / \mathrm{E}^{3}$ x $100 \mathrm{e}$ para classificar o tipo de crescimento intra-uterino, utilizaram-se os valores de 2,25 e 3,10 dos percentis 10 e 90 de Lubchenco. Classificou-se como CIUR assimétrico aquele com IP $<2,25$, e como simétrico quando o IP estivesse entre 2,25 - 3,10. 
Resultados: A média do IP foi semelhante entre os grupos estudados: PEL, PEG e eclâmpsia $(2,60 \pm 0,3)$, tabagiasta $(2,50 \pm 0,4)$, amniorrexe $(2,50 \pm 0,3)$ quando comparadas àquela dos $\mathrm{RN}$ de mulheres hígidas $(2,50$ $\pm 0,3)$. Tomando-se como base o IPR, a taxa de CIUR foi de $95,6 \%(218 / 228)$ nos RN de PEL, 92,3\% (48/52) de PEG e $88 \%(22 / 25)$ de eclâmpsia. No grupo dos RN de tabagistas e de amniorrexe essa taxa foi de 99\% (245/ $247)$ e $99,2 \%(135 / 136)$ respectivamente, valores semelhantes àquele dos RN de mulheres hígidas 98\% (2011/2053). Considerando o IP e o peso ao nascer envolvendo todos os RN dos grupos estudados, verificou-se que $54,5 \%(54 / 99)$ com baixo peso $(<2500 \mathrm{~g})$, $25,9 \%(184 / 711)$ com peso insuficiente (2501 - $3000 \mathrm{~g})$ e $7,7 \%$ (146/1908) com peso adequado (3001 - $4000 \mathrm{~g})$ apresentaram CIUR do tipo assimétrico, enquanto que $45,5 \%$ (45/49) com baixo peso, 73,5\% (523/711) com peso insuficiente, $89,6 \%(1710 / 1908)$ com peso adequado e $73,9 \%(17 / 23)$ com peso excessivo (> $4000 \mathrm{~g})$ foram classificados como portadores de CIUR simétrico. Por outro lado apenas 0,6\% (4/711) dos RN com peso insuficiente, $2,7 \%$ (52/1908) com peso adequado e $26,1 \%(6 / 23)$ com peso excessivo não apresentaram nenhum tipo de CIUR. A comparação entre os RN com pesos adequados e excessivos com e sem CIUR, mostrou diferença estatisticamente significante $(\mathrm{p}<$ $0,0001)$. A associação entre o CIUR e a morbidade neonatal precoce, mostrou que 82,8\% (318/384) dos RN classificados como assimétricos eram hígidos, bem como 84,6\% (1941/2295) dos simétricos, independente dos grupos aos quais pertenciam os RN. A morbidade neonatal precoce manifestou-se representada pelos seguintes parâmetros: taquipnéia transitória que ocorreu entre os RN assimétricos e simétricos em $8,3 \%$ $(32 / 384)$ e $7,2 \%(166 / 2295)$ respectivamente. Constatou-se asfixia no período neonatal precoce em $5,7 \%$ (22/384) dos RN assimétricos e em 5,8\% (132/2295) dos simétricos. A taxa de infecção também foi semelhante entre os dois grupos: 2,6\% (10/384) no assimétrico e 1,7\% (40/2295) no simétrico. Salientese que as variáveis representativas da morbidade neonatal acima citadas, não mostraram diferenças estatisticamente significantes $(\mathrm{p}>0,05)$ quando os dois grupos foram comparados. Em relação à mortalidade neonatal precoce (MNP), ela foi extremamente baixa, sendo $0,3 \%(1 / 384)$ no grupo assimétrico e $0,3 \%$ (6/ 2295) no simétrico. Saliente-se que desses seis óbitos, 4 ocorreram entre os RN de mulheres hígidas.

Conclusão: O presente estudo mostrou que isoladamente, o IPR não foi eficiente para predizer o CIUR na nossa população, visto que, um elevado contingente de RN com pesos adequados e pesos excessivos foram classificados como portadores de retardo de crescimento do tipo simétrico.

Palavras-chave: Crescimento intra-uterino retardado. Mortalidade perinatal.

\section{COMUNICADO AOS ASSOCIADOS E LEITORES}

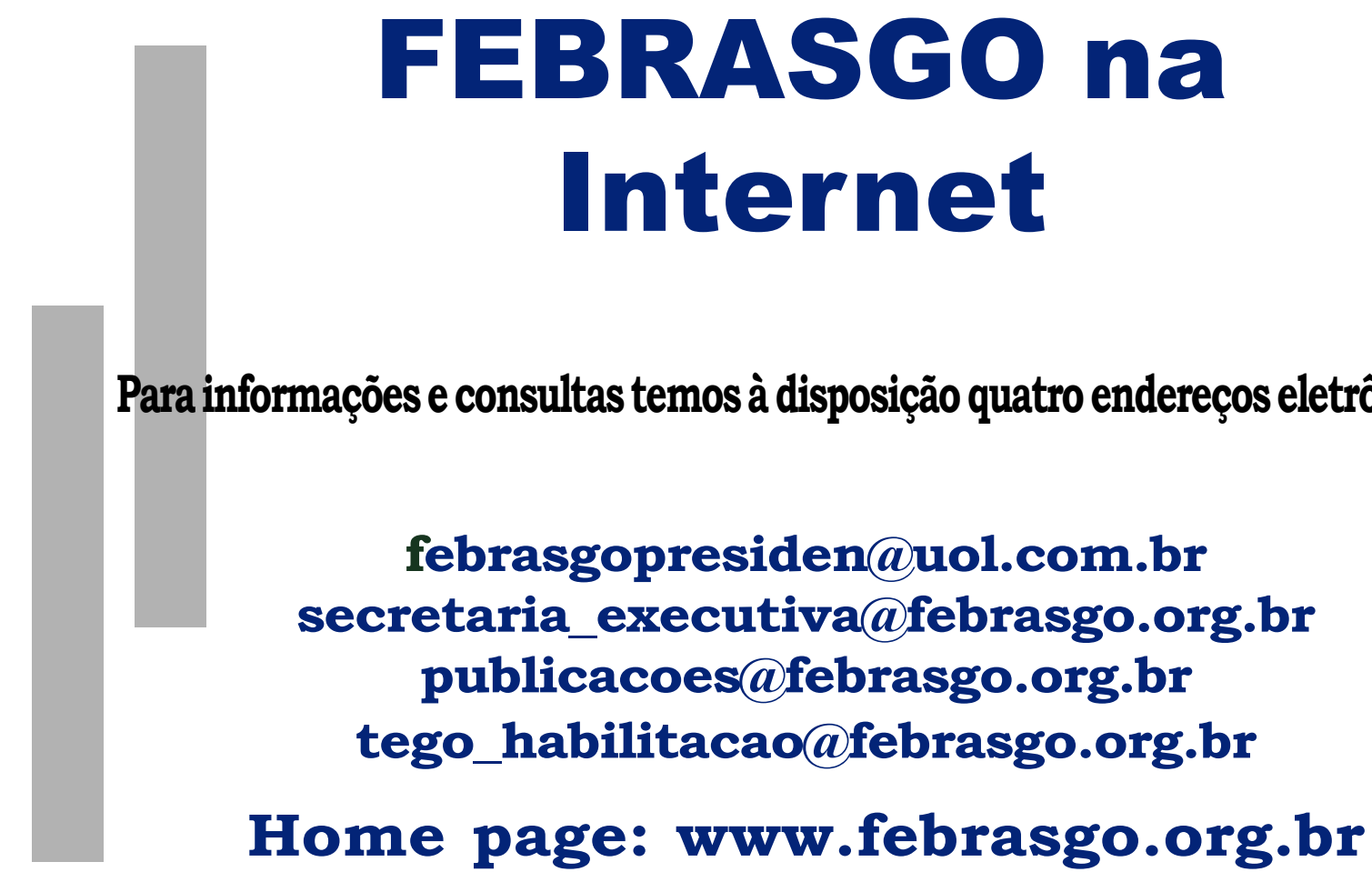

